\title{
Front Matter: Volume 9748
}

, "Front Matter: Volume 9748," Proc. SPIE 9748, Gallium Nitride Materials and Devices XI, 974801 (18 July 2016); doi: 10.1117/12.2239452

SPIE. Event: SPIE OPTO, 2016, San Francisco, California, United States 


\title{
PROCEEDINGS OF SPIE
}

\section{Gallium Nitride Materials and Devices XI}

\author{
Jen-Inn Chyi \\ Hiroshi Fujioka \\ Hadis Morkoç \\ Yasushi Nanishi \\ Ulrich T. Schwarz \\ Jong-In Shim \\ Editors
}

15-18 February 2016

San Francisco, California, United States

Sponsored and Published by

SPIE 
The papers in this volume were part of the technical conference cited on the cover and title page. Papers were selected and subject to review by the editors and conference program committee. Some conference presentations may not be available for publication. Additional papers and presentation recordings may be available online in the SPIE Digital Library at SPIEDigitallibrary.org.

The papers reflect the work and thoughts of the authors and are published herein as submitted. The publisher is not responsible for the validity of the information or for any outcomes resulting from reliance thereon.

Please use the following format to cite material from these proceedings:

Author(s), "Title of Paper," in Gallium Nitride Materials and Devices XI, edited by Jen-Inn Chyi, Hiroshi Fujioka, Hadis Morkoç, Yasushi Nanishi, Ulrich T. Schwarz, Jong-In Shim, Proceedings of SPIE Vol. 9748 (SPIE, Bellingham, WA, 2016) Article CID Number.

ISSN: 0277-786X

ISSN: 1996-756X (electronic)

ISBN: 9781628419832

Published by

SPIE

P.O. Box 10, Bellingham, Washington 98227-0010 USA

Telephone +1 3606763290 (Pacific Time) · Fax +1 3606471445

SPIE.org

Copyright (C) 2016, Society of Photo-Optical Instrumentation Engineers.

Copying of material in this book for internal or personal use, or for the internal or personal use of specific clients, beyond the fair use provisions granted by the U.S. Copyright Law is authorized by SPIE subject to payment of copying fees. The Transactional Reporting Service base fee for this volume is $\$ 18.00$ per article (or portion thereof), which should be paid directly to the Copyright Clearance Center (CCC), 222 Rosewood Drive, Danvers, MA 01923. Payment may also be made electronically through CCC Online at copyright.com. Other copying for republication, resale, advertising or promotion, or any form of systematic or multiple reproduction of any material in this book is prohibited except with permission in writing from the publisher. The CCC fee code is 0277-786X/16/\$18.00.

Printed in the United States of America.

Publication of record for individual papers is online in the SPIE Digital Library.

\section{SPIE. DIGITAL}

Paper Numbering: Proceedings of SPIE follow an e-First publication model. A unique citation identifier (CID) number is assigned to each article at the time of publication. Utilization of CIDs allows articles to be fully citable as soon as they are published online, and connects the same identifier to all online and print versions of the publication. SPIE uses a six-digit CID article numbering system structured as follows:

- The first four digits correspond to the SPIE volume number.

- The last two digits indicate publication order within the volume using a Base 36 numbering system employing both numerals and letters. These two-number sets start with 00, 01, 02, 03, 04, $05,06,07,08,09,0 A, 0 B \ldots$ OZ, followed by 10-1Z, 20-2Z, etc. The CID Number appears on each page of the manuscript. 


\title{
Contents
}

\author{
vii Authors \\ ix Conference Committee
}

BN II

974805 Heteroepitaxial growth of cubic boron nitride films on diamond(001) substrates and their $n$ type doping (Invited Paper) [9748-4]

GROWTH I

974809 HVPE-GaN growth on GaN-based advanced substrates by Smart Cut (Invited Paper) [9748-8]

\section{GROWTH II}

9748 OK Optical and crystal quality improvement in green emitting InxGa1-xN multi-quantum wells through optimization of MOCVD growth [9748-18]

\section{CHARACTERIZATION I}

$9748 \mathrm{OL}$ Quantum well intermixing and radiation effects in InGaN/GaN multi quantum wells (Invited Paper) [9748-19]

\section{CHARACTERIZATION II}

$9748 \mathrm{OQ}$ New directions in GaN photonics (Invited Paper) [9748-24]

9748 OS Analysis of radiative and non-radiative lifetimes in GaN using accurate internal-quantumefficiency values estimated by simultaneous photoluminescence and photo-acoustic measurements [9748-27]

\section{CHARACTERIZATION III}

9748 OU Approaches to highly efficient UV emitters based on AIGaN quantum wells (Invited Paper) [9748-29]

9748 OW Influence of vacancies on GaN/AIN interface characteristics [9748-31] 
9748 OY Interface control technologies for high-power GaN transistors: Self-stopping etching of $p$ GaN layers utilizing electrochemical reactions (Invited Paper) [9748-33]

$97480 Z$ Monolithic enhancement-mode and depletion-mode GaN-based MOSHEMTs (Invited Paper) [9748-34]

LASERS I

974815 Development for ultraviolet vertical cavity surface emitting lasers [9748-40]

LASERS II

974817 Continuous wave operation of high power GaN-based blue vertical-cavity surfaceemitting lasers using epitaxial lateral overgrowth (Invited Paper) [9748-42]

974818 Optical-loss suppressed InGaN laser diodes using undoped thick waveguide structure (Invited Paper) [9748-43]

974819 AIGalnN laser diode technology for systems applications [9748-44]

9748 1A InGaN/GaN DFB laser diodes at $434 \mathbf{n m}$ with deeply etched sidewall gratings [9748-45]

9748 1B Comparison of nonpolar III-nitride vertical-cavity surface-emitting lasers with tunnel junction and ITO intracavity contacts [9748-46]

\section{NANOSTRUCTURES}

9748 1E Linearly polarized single photons from small site-controlled GaN nanowire quantum dots [9748-49]

$97481 G \quad$ Strain engineered high reflectivity DBRs in the deep UV [9748-51]

LEDS I

$97481 \mathrm{~J}$ Deep ultraviolet light-emitting and laser diodes (Invited Paper) [9748-54]

974810 Influence of the LED heterostructure on the degradation behavior of (InAIGa)N-based UV-B LEDs [9748-59]

LEDS II

9748 is Tunable, full-color nanowire light emitting diode arrays monolithically integrated on Si and sapphire [9748-63]

iv 
$97481 \mathrm{U} \quad$ High-efficiency blue LEDs with thin AIGaN interlayers in InGaN/GaN MQWs grown on Si (111) substrates (Invited Paper) [9748-65]

9748 1V GaN-based superluminescent diodes with long lifetime [9748-66]

9748 1W Determination of internal quantum efficiency in GaN by simultaneous measurements of photoluminescence and photo-acoustic signals [9748-67]

974812 Designing optically pumped InGaN quantum wells with long wavelength emission for a phosphor-free device with polarized white-light emission [9748-71]

974820 Hierarchical growth of GaN nanowires for light emitting diode applications [9748-72]

POSTER SESSION

974825 Enhancement of indium incorporation to InGaN MQWs on AIN/GaN periodic multilayers [9748-78]

974826 Exciton localization in (11-22)-oriented semi-polar InGaN multiple quantum wells [9748-79]

974827 Wurtzite/zinc-blende electronic-band alignment in basal-plane stacking faults in semipolar GaN [9748-80]

974828 Optical investigation of microscopic defect distribution in semi-polar (1-101 and 11-22) InGaN light-emitting diodes [9748-81] 
Proc. of SPIE Vol. $9748974801-6$

Downloaded From: https://www.spiedigitallibrary.org/conference-proceedings-of-spie on 26 Apr 2023 Terms of Use: https://www.spiedigitallibrary.org/terms-of-use 


\title{
Authors
}

Numbers in the index correspond to the last two digits of the six-digit citation identifier (CID) article numbering system used in Proceedings of SPIE. The first four digits reflect the volume number. Base 36 numbering is employed for the last two digits and indicates the order of articles within the volume. Numbers start with 00, 01, 02, 03, 04, 05, 06, 07, 08, 09, OA, OB...0Z, followed by 10-12, 20-2Z, etc.

\author{
Akazawa, Masamichi, OY \\ Alves, E., OL \\ Alves, L. C., OL \\ Amilusik, Mikolaj, 09 \\ Andrade, Nicolas, 28 \\ Arakawa, Yasuhiko, $1 \mathrm{E}$ \\ Arif, Ronald A., OK \\ Arita, Munetaka, 1E \\ Armour, Eric A., OK \\ Asif, Fatima, $1 \mathrm{~J}$ \\ Avrutin, Vitaliy, 25, 26, 27, 28 \\ Ben Sedrine, N., OL \\ Berkman, Erkan A., OK \\ Bezyazychnaya, Tatyana V., OW \\ Bobea, M., $1 G$ \\ Boćkowski, Michal, 09, 19 \\ Bryan, I., 1G \\ Carlin, J.-F., IV \\ Castiglia, A., IV \\ Caulmilone, Raphael, 09 \\ Chang, Jhe-Hao, $\mathrm{OZ}$ \\ Choi, Kihyun, $1 \mathrm{E}$ \\ Cohen, D. A., 1B \\ Collazo, R., $1 G$ \\ Correia, M. R., OL \\ Czernecki, R., 19 \\ Das, Saikat, 25, 26, 27, 28 \\ DenBaars, Steven P., 1B, 1 Z \\ Detchprohm, Theeradetch, 15 \\ Docherty, Kevin E., 1A \\ Duelk, M., IV \\ Dupuis, Russell D., 15 \\ Edamoto, Masaaki, OY \\ Einfeldt, Sven, 10 \\ Enslin, Johannes, 10 \\ Farrell, Robert M., 12 \\ Fijalkowski, Michal, 09 \\ Franke, A., IG \\ Freitas, A., OL \\ Funato, Mitsuru, OU \\ Fuutagawa, Noriyuki, 17 \\ Gerhold, M., $1 G$ \\ Gil, Bernard, 26 \\ Glaab, Johannes, 10 \\ Grandjean, N., 1V \\ Grzanka, Ewa, 09 \\ Grzegory, Izabella, 09 \\ Guiot, Eric, 09 \\ Hafiz, Shopan, 25, 27, 28
}

Hagino, Hiroyuki, 18

Hamaguchi, Tatsushi, 17

Han, Jung, $O Q$

Hashizume, Tamotsu, OY

Hernandez-Balderrama, L., IG

Hoffmann, M. P., $1 G$

Holmes, Mark J., $1 \mathrm{E}$

Ichikawa, Shuhei, ou

Imafuji, Osamu, 18

Ito, Toshihide, $1 \mathrm{U}$

Iwinska, Malgorzata, 09

Izumi, Shouichiro, 17

Izyumskaya, Natalia, 25, 26, 27, 28

Kabanau, Dzmitri M., OW

Kaess, F., $1 G$

Kako, Satoshi, $1 \mathrm{E}$

Kao, Tsung-Ting, 15

Katayama, Takuma, 18

Kawaguchi, Masao, 18

Kawakami, K., OS, 1 W

Kawakami, Yoichi, OU

Kelly, Anthony E., 19, 1 A

Khan, Asif, $1 \mathrm{~J}$

Kimura, Shigeya, $1 \mathrm{U}$

Kirste, R., $1 G$

Knaver, Arne, 10

Kneissl, Michael, 10

Kolbe, Tim, 10

Kowsz, Stacy J., 1 Z

Kucharski, R., 19

Kueller, Viola, 10

Kuhn, Christian, 10

Kumazaki, Yusuke, OY

Lebiadok, Yahor V., OW

Lee, Cheul-Ro, 20

Lee, Ching-Ting, $0 z$

Lee, Soo Min, OK

Leitão, M. P., OL

Leonard, J. T., 1B

Leszczyński, M., 19

Litwin-Staszewska, Elzbieta, 09

Liu, Yuh-Shiuan, 15

Lobo Ploch, Neysha, 10

Lorenz, K., OL

Lourenço, M. B., OL

Lucznik, Boleslaw, 09

Margalith, T., 1B

Marona, L., 19

Matuschek, N., IV 
Mehnke, Frank, 10

Mehta, Karan, 15

Meredith, Wyn, 1A

Mi, Zetian, 1S

Monavarian, Morteza, 25, 26, 27, 28

Monteiro, T., OL

Morkoç, Hadis, 25, 26, 27, 28

Mrotzek, Tobias, 09

Muhtadi, Sakib, $1 \mathrm{~J}$

Murayama, Masahiro, 17

Najda, S. P., 19

Nakamura, Shuji, 1B, 1 Z

Nakano, T., OS, IW

Narui, Hironobu, 17

Navamathavan, R., 20

$\mathrm{Ng}, \mathrm{T}$. K., 1B

Nguyen, Hieu P. T., $1 S$

Nowakowska-Siwinska, Anna, 09

Nozaki, Shinichiro, 18

Nunove, Shinya, $1 \mathrm{U}$

Obheroi, Sonika, 20

Odedina, Opeoluwa, 1A

Okada, Aoi, $1 \mathrm{U}$

Ooi, B. S., 1B

Özgür, Ümit, 25, 26, 27, 28

Papasouliotis, George D., OK

Park, Sunghyun, $0 Q$

Peres, M., OL

Perlin, P., 19

Ponce, Fernando A., 15

Pynn, Christopher D., 1 Z

$\mathrm{Ra}$, Yong-Ho, $1 \mathrm{~S}$

Ra, Yong-Ho, 20

Raj, Rishabh, 20

Ramos, Frank, OK

Rass, Jens, 10

Redondo-Cubero, A., OL

Rezzonico, R., $1 \mathrm{~V}$

Rodrigues, J., OL

Rosales, Daniel, 26

Rossetti, M., IV

Ryabtsev, Gennadii I., OW

Sato, Taketomo, OY

Seiss, Martin, 09

Sequeira, M. C., OL

Shen, C., 1B

Shen, Shyh-Chiang, 15

Shih, Ishiang, is

Sitar, Z., 1G

Slight, Thomas J., 1 A

Sochacki, Tomasz, 09

Speck, James S., 1B, 1 Z

Stoelmacker, Christoph, 10

Suski, T., 19

Takigawa, Shinichi, 18

Tanaka, Tsuyoshi, 18

Targowski, G., 19

Tseng, Chun-Yen, $\mathrm{OZ}$

Tucker, Eric, OK
Tweedie, J., $1 G$

Uesugi, Kenjiro, $1 \mathrm{U}$

Vélez, C., IV

Wang, Renjie, is

Washiyama, S., $1 G$

Watson, S., 19

Wernicke, Tim, 10

Weyers, Markus, 10

Wisniewski, P., 19

Wu, Feng, $1 \mathrm{Z}$

Wu, Yuanpeng, is

Xie, Hongen, 15

Xiong, Kanglin, $\mathrm{OQ}$

Yamaguchi, A. A., OS, IW

Yin, Hong, 05

Yoder, P. Douglas, 15

Yonkee, B. P., 1B

Yoshida, Hisashi, $1 \mathrm{U}$

Young, E. C., 1B

Yuan, Ge, OQ

Zhang, Cheng, $O Q$

Zhang, Fan, 28

Zhao, Songrui, is

Zhuravlev, Konstantin S., OW

viii

Proc. of SPIE Vol. $9748974801-8$ 


\section{Conference Committee}

Symposium Chairs

Jean-Emmanuel Broquin, IMEP-LAHC (France)

Shibin Jiang, AdValue Photonics, Inc. (United States)

Symposium Co-chairs

David L. Andrews, University of East Anglia (United Kingdom)

Alexei L. Glebov, OptiGrate Corporation (United States)

Program Track Chair

James G. Grote, Air Force Research Laboratory (United States)

Conference Chairs

Jen-Inn Chyi, National Central University (Taiwan)

Hiroshi Fujioka, The University of Tokyo (Japan)

Hadis Morkoç, Virginia Commonwealth University (United States)

Conference Co-chairs

Yasushi Nanishi, Ritsumeikan University (Japan)

Ulrich T. Schwarz, IMTEK, Universität Freiburg (Germany)

Jong-In Shim, Hanyang University (Korea, Republic of)

Conference Program Committee

Frank Bertram, Otto-von-Guericke-Universität Magdeburg (Germany)

Michal Bockowski, Institute of High Pressure Physics (Poland)

Enrique Calleja, Universidad Politécnica de Madrid (Spain)

Shigefusa F. Chichibu, Tohoku University (Japan)

Bernard Gil, Université Montpellier 2 (France)

Nicolas Grandjean, Ecole Polytechnique Fédérale de Lausanne

(Switzerland)

Hideki Hirayama, RIKEN (Japan)

Ray-Hua Horng, National Chung Hsing University (Taiwan)

Stacia Keller, University of California, Santa Barbara (United States)

Michael Kneissl, Technische Universität Berlin (Germany)

Hao-Chung Kuo, National Chiao Tung University (Taiwan)

Masaaki Kuzuhara, University of Fukui (Japan)

Koh Matsumoto, Taiyo Nippon Sanso Corporation (Japan)

Hideto Miyake, Mie University (Japan)

Eva Monroy, CEA Grenoble (France) 
Yong-Tae Moon, LG Electronics Inc. (Korea, Republic of)

Ki-Bum Nam, Seoul Semiconductor (Korea, Republic of)

Ümit Özgür, Virginia Commonwealth University (United States)

Joachim Piprek, NUSOD Institute LLC (United States)

Tae-Yeon Seong, Korea University (Korea, Republic of)

Chih-Chung Yang, National Taiwan University (Taiwan)

Euijoon Yoon, Seoul National University (Korea, Republic of)

Enrico Zanoni, Università degli Studi di Padova (Italy)

\section{Session Chairs}

$1 \mathrm{BN}$ I

Hadis Morkoç, Virginia Commonwealth University (United States)

2 BN II

Hadis Morkoç, Virginia Commonwealth University (United States)

3 Growth I

Yasushi Nanishi, Ritsumeikan University (Japan)

4 Growth II

Michal Bockowski, Institute of High Pressure Physics (Poland)

5 Characterization I

Hiroshi Fujioka, The University of Tokyo (Japan)

6 Characterization II

Frank Bertram, Otto-von-Guericke- Universität Magdeburg (Germany)

7 Characterization III

Robert Martin, University of Strathclyde (United Kingdom)

8 Electron Devices

Jen-Inn Chyi, National Central University (Taiwan)

9 Lasers I

Ulrich T. Schwarz, Technische Universität Chemnitz (Germany)

10 Lasers II

Hideki Hirayama, RIKEN (Japan)

11 Nanostructures

Elison Matioli, Ecole Polytechnique Fédérale de Lausanne

(Switzerland)

12 LEDs I

Jong-In Shim, Hanyang University (Korea, Republic of) 
13 LEDs II

Asif M. Khan, University of South Carolina (United States)

14 LEDs III

Hadis Morkoç, Virginia Commonwealth University (United States)

Proc. of SPIE Vol. $9748974801-11$

Downloaded From: https://www.spiedigitallibrary.org/conference-proceedings-of-spie on 26 Apr 2023 Terms of Use: https://www.spiedigitallibrary.org/terms-of-use 
Proc. of SPIE Vol. 9748 974801-12

Downloaded From: https://www.spiedigitallibrary.org/conference-proceedings-of-spie on 26 Apr 2023 Terms of Use: https://www.spiedigitallibrary.org/terms-of-use 